\title{
Fully packed loop configurations: polynomiality and nested arches
}

\author{
Florian Aigner $1 \rrbracket$ \\ ${ }^{1}$ Fakultät für Mathematik, Universität Wien, Oskar-Morgenstern-Platz 1, 1090 Vienna, Austria
}

\begin{abstract}
This extended abstract proves that the number of fully packed loop configurations whose link pattern consists of two noncrossing matchings separated by $m$ nested arches is a polynomial in $m$. This was conjectured by Zuber (2004) and for large values of $m$ proved by Caselli et al. (2004).

Résumé. Dans cet article nous prouvons que le nombre de configurations de boucles compactes dont le motif de liaison consiste en deux couplages non-croisés séparés par $m$ arcs emboîtés est un polynôme en $m$. Ce résultat avait été conjecturé par Zuber (2004) et prouvé pour les grandes valeurs de $m$ par Caselli et al. (2004).
\end{abstract}

Keywords. Fully packed loop configurations, wheel polynomials, nested arches

\section{Introduction}

Razumov and Stroganov conjectured in [5] a relation between fully packed loop configurations (short $F P L s)$ and the ground state vector in the $O(1)$ loop model. This conjectural connection made it possible to come up with many new conjectures concerning FPLs with certain link patterns, see $e$. g. [11]. In this paper we will prove the following

Theorem 1.1 ([11, Conjecture 7]) For noncrossing matchings $\pi \in N C_{n}$ and $\pi^{\prime} \in N C_{n^{\prime}}$ and an integer $m$ the number of FPLs with link pattern $(\pi)_{m} \pi^{\prime}$ is a polynomial in $m$ of degree $|\lambda(\pi)|+\left|\lambda\left(\pi^{\prime}\right)\right|$ with leading coefficient $\frac{\operatorname{dim}(\lambda(\pi)) \operatorname{dim}\left(\lambda\left(\pi^{\prime}\right)\right)}{|\lambda(\pi)| !\left|\lambda\left(\pi^{\prime}\right)\right| !}$.

The special case for only one matching was completely shown in [2][Theorem 4.2], whereas the general case was proven only for large values of $m$, see [2][Theorem 6.7]. With the proof of the RazumovStroganov Conjecture in [1], it was possible to use new methods - namely the theory of wheel polynomials which was developed in [2], [9] - for solving open problems concerning FPLs. Using integral wheel polynomials, Fonseca and Zinn-Justin reproved in [4] Theorem 1.1 for the special case of one matching. By considering another well known family of wheel polynomials we will be able to prove Theorem 1.1 .

\footnotetext{
$\dagger$ Email: florian.aigner@univie.ac.at.

Research supported by the Austrian Science Fund (FWF), START grant Y463.

1365-8050 @ 2016 Discrete Mathematics and Theoretical Computer Science (DMTCS), Nancy, France
} 


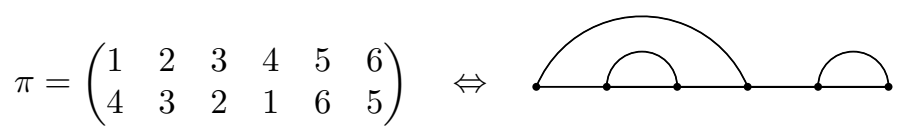

Fig. 1: An example for a noncrossing matching and its graphical representation.

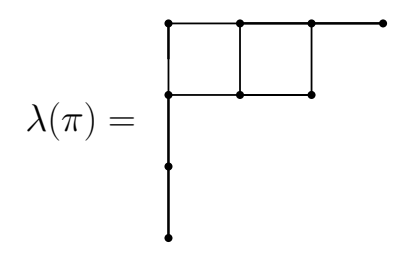

Fig. 2: The corresponding Young diagram to the noncrossing matching of the previous example.

\section{Preliminaries}

\subsection{Noncrossing matchings}

A noncrossing matching $\pi$ of size $n$ is a derangement $\pi \in S_{2 n}$ with $\pi^{2}=I d_{2 n}$ such that there exist no integers $1 \leq a<b<c<d \leq 2 n$ with $\pi(a)=c$ and $\pi(b)=d$. We denote by $N C_{n}$ the set of noncrossing matchings of size $n$. The number of noncrossing matchings of size $n$ is given by the $n$-th Catalan number $C_{n}=\frac{1}{n+1}\left(\begin{array}{c}2 n \\ n\end{array}\right)$. A noncrossing matching $\pi$ can be represented graphically by $2 n$ dots which are labeled from 1 to $2 n$ and two dots are linked by an arc iff their labels are mapped onto each other by $\pi$, see Figure 1. In the following we do not want to distinguish between the graphical representation of a noncrossing matching $\pi$ and $\pi$ itself.

A Young diagram is a finite collection of boxes, arranged in left-justified rows and weakly decreasing row-length from top to bottom (English notation). The noncrossing matchings of size $n$ are in bijection with the Young diagrams for which the $i$-th row from the top consists of at most $n-i$ boxes. We construct for a noncrossing matching $\pi$ its corresponding Young diagram $\lambda(\pi)$ by reading its parenthesis word from left to right and make an up-step if an opening bracket is read and a right-step if a closing bracket is read. The Young diagram $\lambda(\pi)$ is then the area enclosed by this path and the path taking $n$ consecutive up-steps followed by $n$ consecutive right-steps, see Figure 2. To keep in mind which size the noncrossing matching $\pi$ with corresponding Young diagram $\lambda(\pi)$ has and for reasons which will be clear later, we will always draw these $n$ consecutive up- / right-steps we needed in the above construction when the Young diagram is depicted Since Young diagrams are in bijection with integer partitions, noncrossing matchings of size $n$ are in bijection to integer partitions where the $i$-th summand is less or equal than $n-i$. The bijection between Young diagrams and integer partitions is in this work given by setting the $i$-th summand of a partition equal to the number of boxes of the $i$-th row from the top of a partition. For a Young diagram $\lambda$ we denote with $|\lambda|$ the number of its boxes.

Let $\pi, \sigma$ be noncrossing matchings of size $n$ or $n^{\prime}$ respectively and $m$ a positive integer. We denote by $(\pi)_{m}$ the noncrossing matching of size $n+m$ which is obtained by putting $m$ nested arches around $\pi$ and by ()$^{m}$ the noncrossing matching which is made out of $m$ consecutive small arches. Further we denote by $\pi \sigma$ the concatenation of $\pi$ and $\sigma$, see Figure 3 . 

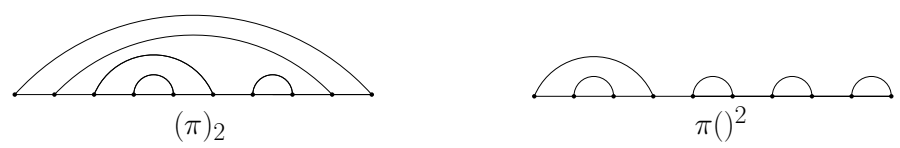

Fig. 3: This figure shows the noncrossing matchings $(\pi)_{2}$ and $\pi()^{2}$, where $\pi$ is as in the above example.

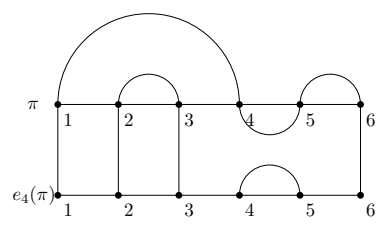

Fig. 4: This is an example for the calculation of $e_{4}(\pi)$ where $\pi$ is the noncrossing matching of the previous examples.

Let $k$ be the index such that $\pi(k)=2 n$. Then define $\rho(\pi)$ as the rotation of $\pi$ given by $\rho(\pi)(i)=$ $\pi(i-1)+1$ for $1<i \leq 2 n$ and $\rho(\pi)(1)=k+1$. For $1 \leq j \leq 2 n$ define $e_{j}: N C_{n} \rightarrow N C_{n}$ as the map

$$
e_{j}(\pi)(k)=\left\{\begin{array}{ll}
j+1 & k=j \\
j & k=j+1 \\
\pi(j+1) & k=\pi(j) \\
\pi(j) & k=\pi(j+1) \\
\pi(k) & \text { otherwise }
\end{array},\right.
$$

where $j+1$ is replaced by 1 if $j=2 n$. It is easy to show that $\rho(\pi)$ and $e_{j}(\pi)$ for $1 \leq j \leq 2 n$ are again noncrossing matchings of size $n$. For a graphical representation of $e_{j}$ see Figure 4

We can define a partial order on the set $N C_{n}$ of all noncrossing matchings of size $n$ by defining $\sigma \leq \pi$ for two elements $\sigma, \pi \in N C_{n}$ if the Young diagram $\lambda(\sigma)$ is contained in $\lambda(\pi)$. For $2 \leq j \leq 2 n-2$ we denote $\sigma \nearrow_{j} \pi$ if $\lambda(\pi)$ is obtained from $\lambda(\sigma)$ by adding one box with top left corner on the $j$-th south-east diagonal, where these diagonals are counted as in Figure 5 This notion is obviously a refinement of the above defined partial order.


Fig. 5: The matchings $\pi, \sigma$ satisfy the refined order $\pi \nearrow_{3} \sigma$. 

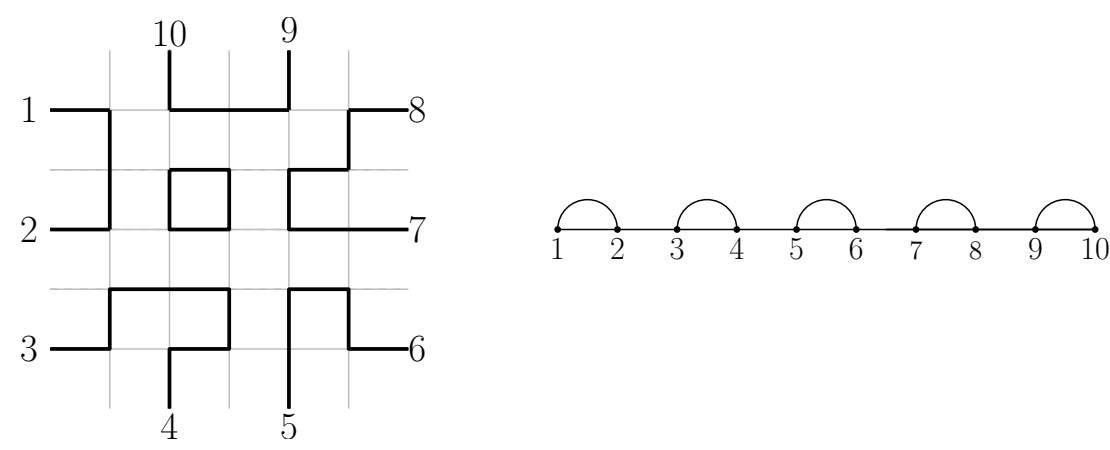

Fig. 6: An example of a FPL of size 5 and its link pattern.

\subsection{Fully packed loop configurations}

A fully packed loop configuration (or short $F P L$ ) $F$ of size $n$ is a subgraph of the $n \times n$ grid with $n$ external edges on every side with the following two properties:

1. F contains all vertices of the $n \times n$ grid and every vertex of $\mathrm{F}$ has degree 2 .

2. $F$ contains every other external edge, beginning with the topmost at the left side.

We enumerate the external edges in a FPL counter-clockwise with 1 up to $2 n$, see Figure 6 A FPL consists out of paths which do not cross each other, the closed ones are called Loops. We can assign to every FPL $F$ a noncrossing matching $\pi(F)$ by setting $\pi(F)(i)$ the label of the external edge which is connected to the $i$-th external edge for $1 \leq i \leq 2 n$, and call $\pi(F)$ the link pattern of $F$.

It is well known that there exists a bijection between all FPLs of size $n$ and all Alternating Sign Matrices (or short $A S M s$ ) of size $n$, see for example [11]. Hence the number $A_{n}$ of all FPLs of size $n$ is equals to the number $A_{n}$ of ASMs of size $n$, see [8]

$$
A_{n}=\prod_{i=1}^{n} \frac{(3 i-2) !}{(n+i-1) !} .
$$

Let $\pi$ be a noncrossing matching of size $n$, we denote with $A_{\pi}$ the number of all FPLs of size $n$ with link pattern $\pi$. In [7] it was shown that the number of FPLs with link pattern $\pi$ is invariant under the action of the dihedral group $D_{n}$ onto the noncrossing matchings of size $n$.

\subsection{Wheel polynomials}

We will introduce in this section wheel polynomials only succinctly, for a more detailed overview see for example [6, Section 2].

For a positive integer $n$ a polynomial $p \in \mathbb{Q}(q)\left[z_{1}, \ldots, z_{2 n}\right]$ is called wheel polynomial of order $n$ if $p$ is a homogeneous polynomial of degree $n(n-1)$ and satisfies the wheel condition:

$$
p\left(z_{1}, \ldots, z_{2 n}\right)_{\mid q^{4} z_{i}=q^{2} z_{j}=z_{k}}=0,
$$

for all $1 \leq i<j<k \leq 2 n$. Denote with $W_{n}[z]$ the space of all wheel polynomials of order $n$ with coefficients in $\mathbb{Q}(q)$. 
Definition 2.1 Let $n$ be an integer and $k \in\{1, \ldots, 2 n\}$. We define the linear maps $S_{k}, D_{k}: \mathbb{Q}(q)\left[z_{1}, \ldots, z_{2 n}\right] \longrightarrow$ $\mathbb{Q}(q)\left[z_{1}, \ldots, z_{2 n}\right]$ via

$$
\begin{aligned}
S_{k}(f)\left(z_{1}, \ldots, z_{2 n}\right) & :=f\left(z_{1}, \ldots, z_{k-1}, z_{k+1}, z_{k}, z_{k+2}, \ldots, z_{2 n}\right), \\
D_{k}(f)\left(z_{1}, \ldots, z_{2 n}\right) & :=\frac{q z_{k}-q^{-1} z_{k+1}}{z_{k+1}-z_{k}}\left(S_{k}(f)\left(z_{1}, \ldots, z_{2 n}\right)-f\left(z_{1}, \ldots, z_{2 n}\right)\right),
\end{aligned}
$$

for $f \in \mathbb{Q}(q)\left[z_{1}, \ldots, z_{2 n}\right]$ and $2 n+1$ is replaced by 1 for $k=2 n$.

It is not difficult to show the following

Lemma 2.2 1. The space $W_{n}[z]$ of all wheel polynomials of order $n$ is closed under the action of $D_{k}$ for $k \in\{1, \ldots, 2 n\}$.

2. For all $k \in\{1, \ldots, 2 n\}$ and all polynomials $f, g \in \mathbb{Q}(q)\left[z_{1}, \ldots, z_{2 n}\right]$ one has:

$$
D_{k}(f g)=D_{k}(f) S_{k}(g)+f D_{k}(g) .
$$

This product rule can also be stated more generally for an integer $m$ and $f_{i} \in \mathbb{Q}(q)\left[z_{1}, \ldots, z_{2 n}\right]$ for $1 \leq i \leq m$ :

$$
D_{k}\left(\prod_{i=1}^{m} f_{i}\right)=\sum_{i=1}^{m}\left(\prod_{j=1}^{i-1} f_{j} \times D_{k}\left(f_{i}\right) \times S_{k}\left(\prod_{j=i+1}^{m} f_{j}\right)\right) .
$$

The next theorem describes a $\mathbb{Q}(q)$-basis of the space $W_{n}[z]$ of all wheel polynomials of order $n$ and a recursion to calculate them. However the most important result for us is that we can calculate the number of FPLs with a certain noncrossing matching as link pattern by evaluating the proper element of this basis at $z_{i}=1$ for all $1 \leq i \leq 2 n$. The proofs for the statements can be found in [9. Section 4], whereas the proof of the last statement also requires the Razumov-Stroganov Conjecture, which was proven in [1].

Theorem 2.3 There exists a $\mathbb{Q}(q)$-basis $\left\{\Psi_{\pi} \mid \pi \in N C_{n}\right\}$ of $W_{n}[z]$ indexed by the noncrossing matchings of size $n$ with the following properties:

1. $\Psi_{()_{n}}=\left(q-q^{-1}\right)^{-n(n-1)} \prod_{1 \leq i<j \leq n}\left(q z_{i}-q^{-1} z_{j}\right)\left(q z_{n+i}-q^{-1} z_{n+j}\right)$.

2. $\Psi_{\pi}(z)=D_{j}\left(\Psi_{\sigma}\right)-\sum_{\tau \in e_{j}^{-1}(\sigma) \backslash\{\sigma, \pi\}} \Psi_{\tau}$, if $\sigma \nearrow_{j} \pi$.

3. $\Psi_{\rho^{-1}(\pi)}\left(z_{1}, \ldots, z_{2 n}\right)=\Psi_{\pi}\left(z_{2}, \ldots, z_{2 n}, q^{6} z_{1}\right)$.

4. Set $q=e^{\frac{2 \pi i}{3}}$ a root of unity, then $\Psi_{\pi}(1, \ldots, 1)=A_{\pi}$ holds for all $\pi \in N C_{n}$.

It is easy to prove that the set of preimages of the noncrossing matching $\sigma$ under $e_{j}$ as in the above setting is a subset of $\{\sigma, \pi\} \cup\left\{\tau \in N C_{n}: \tau<\sigma\right\}$. Since noncrossing matchings and Dyck paths are in bijection we can define for all $1 \leq j \leq 2 n$ a map corresponding to $e_{j}$ for Dyck paths. With the explicit description of these maps in [3. Proposition 2.1] it is easy to prove the following

Lemma 2.4 Let $\alpha, \beta$ be noncrossing matchings of size $n$ or $n^{\prime}$ respectively and $m$ an integer. Then

$$
e_{2(n+m)+i}^{-1}\left((\alpha)_{m} \beta\right)=\left\{(\alpha)_{m} \sigma \mid e_{i}(\sigma)=\beta\right\}
$$

for $2 \leq i \leq 2 n^{\prime}-2$. 
Remark 2.5 Let $\pi$ be a noncrossing matching of size $n$ and denote with $\Lambda_{\pi}$ the set of indices such that all boxes of $\lambda(\pi)$ lie on diagonals which are indexed with numbers in $\Lambda_{\pi}$. One can show inductively using the first two statements of the Theorem 2.3. that $\Psi_{\pi}$ is a sum of terms of the form

$$
D_{i_{1}} \circ \cdots \circ D_{i_{m}}\left(\Psi_{()_{n}}\right)
$$

where $m \leq|\lambda(\pi)|$ and $i_{1}, \cdots i_{m} \in \Lambda_{\pi}$. The number of summands and the indices $i_{1}, \ldots, i_{m}$ appearing in the summands depend only on shape of the Young diagram $\lambda(\pi)$. This implies that for any positive integer $m$ the wheel polynomial $\Psi_{(\pi)_{m}}$ has the same presentation as a sum of the above form as $\Psi_{\pi}$ except that $\Psi_{()_{n}}$ is replaced by $\Psi_{()_{n+m}}$ in the summands. Hence this presentation of $\Psi_{(\pi)_{m}}$ as a sum depends only on $\pi$ and therefore independent on $m$.

\section{The proof}

The following notations are only for the sake of brevity:

$$
f(i, j):=\frac{q z_{i}-q^{-1} z_{j}}{q-q^{-1}}, \quad g(i):=\frac{q-q^{-1} z_{i}}{q-q^{-1}}, \quad h(i):=\frac{q z_{i}-q^{-1}}{q-q^{-1}}
$$

for $1 \leq i \neq j \leq 2 n$. One verifies by simple calculation:

Lemma 3.1 For $1 \leq i, j, k \leq 2 n$ and $i \neq j$ one has

1. $D_{k}(f(i, j))= \begin{cases}f(k, k+1) & (i, j)=(k+1, k) \\ -f(k, k+1) & (i, j)=(k, k+1) \\ q f(k, k+1) & i=k ; j \neq k, k+1 \\ -q f(k, k+1) & i=k+1 ; j \neq k, k+1 \\ -q^{-1} f(k, k+1) & j=k ; i \neq k, k+1 \\ q^{-1} f(k, k+1) & j=k+1 ; i \neq k, k+1 \\ 0 & \{i, j\} \cap\{k, k+1\}=\emptyset\end{cases}$

2. $D_{k}(g(i))= \begin{cases}-q^{-1} f(k, k+1) & i=k \\ q^{-1} f(k, k+1) & i=k+1 \\ 0 & \text { otherwise }\end{cases}$

3. $D_{k}(h(i))= \begin{cases}q f(k, k+1) & i=k \\ -q f(k, k+1) & i=k+1 \\ 0 & \text { otherwise }\end{cases}$ 
4. Let $m$ be a positive integer. Then the following holds:

$$
\begin{aligned}
D_{k}\left(f(i, j)^{m}\right) & =D_{k}(f(i, j)) \sum_{l=0}^{m-1} f(i, j)^{l} S_{k}\left(f(i, j)^{m-1-l}\right), \\
D_{k}\left(g(i)^{m}\right) & =D_{k}(g(i)) \sum_{l=0}^{m-1} g(i)^{l} S_{k}\left(g(i)^{m-1-l}\right), \\
D_{k}\left(h(i)^{m}\right) & =D_{k}(h(i)) \sum_{l=0}^{m-1} h(i)^{l} S_{k}\left(h(i)^{m-1-l}\right) .
\end{aligned}
$$

We use the following notation:

$$
P\left(\alpha_{i, j}\left|\beta_{i}\right| \gamma_{i}\right):=\prod_{1 \leq i \neq j \leq 2 n} f(i, j)^{\alpha_{i, j}} \prod_{i=1}^{2 n} g(i)^{\beta_{i}} h(i)^{\gamma_{i}} .
$$

Theorem 3.2 Let $P=P\left(\alpha_{i, j}\left|\beta_{i}\right| \gamma_{i}\right), m$ an integer and $i_{1}, \ldots, i_{m} \in\{1, \ldots, 2 n\}$. Then there exists a polynomial $Q \in \mathbb{Q}(q)\left[y_{1}, \ldots, y_{2 n(2 n+1)}\right]$ of degree at most $m$ such that

$$
\left(D_{i_{1}} \circ \cdots \circ D_{i_{m}}\right)(P)_{\mid z_{1}=\ldots=z_{2 n}=1}=Q\left(\left(\alpha_{i, j}\right),\left(\beta_{i}\right),\left(\gamma_{i}\right)\right) .
$$

The following example is included to sketch the main idea of the proof of the above Theorem and to help the reader in understanding it.

Example 3.3 Let $n=m=i_{m}=1$ Then we can calculate $D_{1}(P)_{z_{1}=z_{2}=1}$ explicitly, where $m, n, i_{m}$ and $P$ are as above. By using Lemma 2.2 and Lemma 3.1 we obtain for $D_{1}(P)$ the following expression:

$$
\begin{aligned}
D_{1}(P)= & D_{1}\left(f(1,2)^{\alpha_{1,2}} f(2,1)^{\alpha_{2,1}} g(1)^{\beta_{1}} g(2)^{\beta_{2}} h(1)^{\gamma_{1}} h(2)^{\gamma_{2}}\right) \\
= & -\sum_{t=1}^{\alpha_{1,2}} f(1,2)^{\alpha_{1,2}+\alpha_{2,1}-t+1} f(2,1)^{t-1} g(1)^{\beta_{2}} g(2)^{\beta_{1}} h(1)^{\gamma_{2}} h(2)^{\gamma_{1}}+ \\
& +\sum_{t=1}^{\alpha_{2,1}} f(1,2)^{\alpha_{1,2}+\alpha_{2,1}-t+1} f(2,1)^{t-1} g(1)^{\beta_{2}} g(2)^{\beta_{1}} h(1)^{\gamma_{2}} h(2)^{\gamma_{1}}+ \\
& -q^{-1} \sum_{t=1}^{\beta_{1}} f(1,2)^{\alpha_{1,2}+1} f(2,1)^{\alpha_{2,1}} g(1)^{\beta_{1}+\beta_{2}-t} g(2)^{t-1} h(1)^{\gamma_{2}} h(2)^{\gamma_{1}}+ \\
& +q^{-1} \sum_{t=1}^{\beta_{2}} f(1,2)^{\alpha_{1,2}+1} f(2,1)^{\alpha_{2,1}} g(1)^{\beta_{1}+\beta_{2}-t} g(2)^{t-1} h(1)^{\gamma_{2}} h(2)^{\gamma_{2}}+ \\
& +q \sum_{t=1}^{\gamma_{1}} f(1,2)^{\alpha_{1,2}+1} f(2,1)^{\alpha_{2,1}} g(1)^{\beta_{1}} g(2)^{\beta_{2}} h(1)^{\gamma_{1}+\gamma_{2}-t} h(2)^{t-1}+ \\
& -q \sum_{t=1}^{\gamma_{2}} f(1,2)^{\alpha_{1,2}+1} f(2,1)^{\alpha_{2,1}} g(1)^{\beta_{1}} g(2)^{\beta_{2}} h(1)^{\gamma_{1}+\gamma_{2}-t} h(2)^{t-1} .
\end{aligned}
$$


If we evaluate this for $z_{1}=z_{2}=1$ we obtain:

$$
D_{1}(P)_{z_{1}=z_{2}=1}=\alpha_{2,1}-\alpha_{1,2}+q^{-1}\left(\beta_{2}-\beta_{1}\right)+q\left(\gamma_{1}-\gamma_{2}\right) .
$$

Proof of Theorem 3.2: We prove the theorem by induction on $m$. The statement is trivial for $m=0$, hence let $m>0$ and set $k:=i_{m}$. We can write

$$
D_{k} P=\sum_{s \in S} a_{s} P_{s}
$$

for a finite set $S$ of indices, $a_{s} \in\left\{ \pm 1, \pm q, \pm q^{-1}\right\}$ and $P_{s}=P\left(\alpha_{i, j ; s}\left|\beta_{i ; s}\right| \gamma_{i ; s}\right)$ for all $s \in S$. Indeed we can use iteratively the second statement of Lemma 2.2 to split the product into a sum. This splitting depends on the order of the factors of the product, the order we will apply is the one which will be used for the calculations in (1) and was used in Example 3.3 Lemma 3.1 implies that every summand is of the form $P_{s}=P\left(\alpha_{i, j ; s}\left|\beta_{i ; s}\right| \gamma_{i ; s}\right)$ and the coefficients $a_{s}$ are as stated above. Therefore we need to calculate

$$
D_{i_{1}} \circ \cdots \circ D_{i_{m-1}}\left(\sum_{s \in S} a_{s} P_{s}\right)=\sum_{s \in S} a_{s} D_{i_{1}} \circ \cdots \circ D_{i_{m-1}}\left(P_{s}\right) .
$$

For this calculation we split $S$ into subsets $A_{i, j}, B_{i}$ and $C_{i}$ with $1 \leq i \neq j \leq 2 n$ by using Lemma2.2 in the following way:

$$
\begin{aligned}
& D_{k} P=D_{k}\left(\prod_{1 \leq i \neq j \leq 2 n} f(i, j)^{\alpha_{i, j}} \prod_{i=1}^{2 n} g(i)^{\beta_{i}} h(i)^{\gamma_{i}}\right) \\
& =\sum_{1 \leq i \neq j \leq 2 n} \prod_{\substack{1 \leq i^{\prime} \neq j^{\prime} \leq 2 n \\
\left(i^{\prime}<i\right) \vee\left(i^{\prime}=i, j^{\prime}<j\right)}} f\left(i^{\prime}, j^{\prime}\right)^{\alpha_{i^{\prime}, j^{\prime}}} \times D_{k}\left(f(i, j)^{\alpha_{i, j}}\right) \times \\
& \times S_{k}\left(\prod_{\substack{1 \leq i^{\prime} i \neq j^{\prime} \leq 2 n \\
\left(i^{\prime}>i\right) \vee\left(i^{\prime}=i, j^{\prime}>j\right)}} f\left(i^{\prime}, j^{\prime}\right)^{\alpha_{i^{\prime}, j^{\prime}}} \prod_{i^{\prime}=1}^{2 n} g\left(i^{\prime}\right)^{\beta_{i^{\prime}}} h\left(i^{\prime}\right)^{\gamma_{i^{\prime}}}\right)+ \\
& +\sum_{i=1}^{2 n} \prod_{1 \leq i^{\prime} \neq j^{\prime} \leq 2 n} f\left(i^{\prime}, j^{\prime}\right)^{\alpha_{i^{\prime}, j^{\prime}}} \prod_{i^{\prime}=1}^{i-1} g\left(i^{\prime}\right)^{\beta_{i^{\prime}}} \times D_{k}\left(g(i)^{\beta_{i}}\right) \times S_{k}\left(\prod_{i^{\prime}=i+1}^{2 n} g\left(i^{\prime}\right)^{\beta_{i^{\prime}}} \prod_{i^{\prime}=1}^{2 n} h\left(i^{\prime}\right)^{\gamma_{i^{\prime}}}\right)+ \\
& +\sum_{i=1}^{2 n} \prod_{1 \leq i^{\prime} \neq j^{\prime} \leq 2 n} f\left(i^{\prime}, j^{\prime}\right)^{\alpha_{i^{\prime}, j^{\prime}}} \prod_{i^{\prime}=1}^{2 n} g\left(i^{\prime}\right)^{\beta_{i^{\prime}}} \prod_{i^{\prime}=1}^{i-1} h\left(i^{\prime}\right)^{\gamma_{i^{\prime}}} \times D_{k}\left(h(i)^{\gamma_{i}}\right) \times S_{k}\left(\prod_{i^{\prime}=i+1}^{2 n} h\left(i^{\prime}\right)^{\gamma_{i^{\prime}}}\right) .
\end{aligned}
$$

We define $A_{i, j} \subset S$ to be the set of all $s \in S$ such that the summands $P_{s}$ originate from calculating the summand in Equation 1 with the factor $D_{k}\left(f(i, j)^{\alpha_{i, j}}\right)$ explicitly - this can be done by using Lemma 3.1. The sets $B_{i}$ and $C_{i}$ are defined analogously where the role of $f(i, j)^{\alpha_{i, j}}$ is replaced by $g(i)^{\beta_{i}}$ or $h(i)^{\gamma_{i}}$ respectively. Hence we can write the set $S$ as the union

$$
S=\bigcup_{1 \leq i \neq j \leq 2 n} A_{i, j} \cup \bigcup_{1 \leq i \leq 2 n} B_{i} \cup \bigcup_{1 \leq i \leq 2 n} C_{i} .
$$


Lemma 3.1 implies $D_{k}(f(i, j))=0$ for $\{i, j\} \cap\{k, k+1\}=\emptyset$ and $D_{k}(g(i))=D_{k}(h(i))=0$ for $i \notin\{k, k+1\}$. Hence the sets $A_{i, j}, B_{i}, C_{i}$ are empty in these cases.

Let $1 \leq i \neq j \leq 2 n$ be fixed with $\{i, j\} \cap\{k, k+1\} \neq \emptyset$ and let $\sigma \in \mathfrak{S}_{2 n}$ be the permutation $\sigma=(k, k+1)$. Set $\Lambda_{i, j}=\left\{\left(i^{\prime}, j^{\prime}\right): 1 \leq i^{\prime} \neq j^{\prime} \leq 2 n,\left(i^{\prime}<i\right) \vee\left(i^{\prime}=i, j^{\prime}<j\right)\right\}$. Then the definition of $A_{i, j}$ and Lemma 3.1 implies for all $\left(i^{\prime}, j^{\prime}\right) \notin\{(i, j),(\sigma(i), \sigma(j)),(k, k+1)\}$ and all $s \in A_{i, j}$ :

$$
\alpha_{i^{\prime}, j^{\prime} ; s}= \begin{cases}\alpha_{i^{\prime}, j^{\prime}} & \left\{i^{\prime}, j^{\prime}\right\} \cap\{k, k+1\}=\emptyset \text { or }\left(i^{\prime}, j^{\prime}\right),\left(\sigma\left(i^{\prime}\right), \sigma\left(j^{\prime}\right)\right) \in \Lambda_{i, j} \\ \left.\alpha_{i^{\prime}, j^{\prime}}+\alpha_{\sigma\left(i^{\prime}\right), \sigma\left(j^{\prime}\right)}\right) & \left\{i^{\prime}, j^{\prime}\right\} \cap\{k, k+1\} \neq \emptyset,\left(i^{\prime}, j^{\prime}\right) \in \Lambda_{i, j},\left(\sigma\left(i^{\prime}\right), \sigma\left(j^{\prime}\right)\right) \notin \Lambda_{i, j} \\ 0 & \left\{i^{\prime}, j^{\prime}\right\} \cap\{k, k+1\} \neq \emptyset,\left(i^{\prime}, j^{\prime}\right) \notin \Lambda_{i, j},\left(\sigma\left(i^{\prime}\right), \sigma\left(j^{\prime}\right)\right) \in \Lambda_{i, j} \\ \alpha_{\sigma\left(i^{\prime}\right), \sigma\left(j^{\prime}\right)} & \left\{i^{\prime}, j^{\prime}\right\} \cap\{k, k+1\} \neq \emptyset,\left(i^{\prime}, j^{\prime}\right),\left(\sigma\left(i^{\prime}\right), \sigma\left(j^{\prime}\right)\right) \notin \Lambda_{i, j}\end{cases}
$$

For $(k, k+1) \notin\{(i, j),(\sigma(i), \sigma(j))\}$ one obtains the parameter $\alpha_{k, k+1 ; s}$ by adding one to the value of $\alpha_{i^{\prime}, j^{\prime}}$ in the above description for $\left(i^{\prime}, j^{\prime}\right)=(k, k+1)$. Further we obtain $\beta_{i^{\prime} ; s}=\beta_{\sigma\left(i^{\prime}\right)}$ and $\gamma_{i^{\prime} ; s}=\gamma_{\sigma\left(i^{\prime}\right)}$ for all $1 \leq i^{\prime} \leq 2 n$ and $s \in A_{i, j}$. By Lemma 3.1 the constant $a_{s}$ is for all $s \in A_{i, j}$ determined by the corresponding constant of $D_{k}(f(i, j))$ and hence not depending on $s$. The last statement of Lemma 3.1 implies that we can list the elements of $A_{i, j}=\left\{s_{1}, \ldots, s_{\alpha_{i, j}}\right\}$ such that we have the following description for the remaining parameters $\alpha_{i, j ; s}, \alpha_{\sigma(i), \sigma(j) ; s}$ :

$$
\begin{array}{r}
\alpha_{i, j ; s_{t}}= \begin{cases}\alpha_{i, j}+\alpha_{j, i}+1-t & i=k, j=k+1 \\
\alpha_{i, j}-t & i=k+1, j=k \\
\alpha_{i, j}+\alpha_{\sigma(i), \sigma(j)}-t & \{i, j\} \cap\{k, k+1\}=k \\
\alpha_{i, j}-t & \{i, j\} \cap\{k, k+1\}=k+1\end{cases} \\
\alpha_{\sigma(i), \sigma(j) ; s_{t}}= \begin{cases}\alpha_{i, j}+\alpha_{j, i}-\alpha_{i, j ; s_{t}} & \{i, j\}=\{k, k+1\} \\
\alpha_{i, j}+\alpha_{\sigma(i), \sigma(j)}-\alpha_{i, j ; s_{t}}-1 & \text { otherwise }\end{cases}
\end{array}
$$

with $1 \leq t \leq \alpha_{i, j}$.

For the sets $B_{i}, C_{i}$ and $i \in\{k, k+1\}$ we have an analogue description as above, whereas the only parameters depending on $s \in B_{i}$ or $s \in C_{i}$ respectively are given in the case of $B_{i}$ by

$$
\beta_{k ; s_{t}}=\beta_{k}+\beta_{k+1}-t, \quad \beta_{k+1 ; s_{t}}=t-1,
$$

with $1 \leq t \leq \beta_{i}$ and in the case of $C_{i}$ by

$$
\gamma_{k ; s_{t}}=\gamma_{k}+\gamma_{k+1}-t, \quad \gamma_{k+1 ; s_{t}}=t-1,
$$

with $1 \leq t \leq \gamma_{i}$.

By induction we know that $D_{i_{1}} \circ \cdots \circ D_{i_{m-1}}\left(P\left(\alpha_{i, j ; s}\left|\beta_{i ; s}\right| \gamma_{i ; s}\right)\right)_{\mid z_{1}=\ldots=z_{2 n}=1}$ is a polynomial $Q^{\prime}$ of degree at most $m-1$ in $\left(\alpha_{i, j ; s}\right),\left(\beta_{i ; s}\right),\left(\gamma_{i ; s}\right)$ for all $s \in S$. The above description implies that if we restrict ourself to $s \in A_{i, j}, s \in B_{i}$ or $s \in C_{i}$ respectively, $a_{s}$ is invariant in $s$, the parameters 
$\left(\alpha_{i, j ; s}\right),\left(\beta_{i ; s}\right),\left(\gamma_{i ; s}\right)$ are linear combinations of $\left(\alpha_{i, j}\right),\left(\beta_{i}\right),\left(\gamma_{i}\right)$ and in two cases also depending linearly on a parameter $t$ which runs from 1 up to the cardinality of the set $A_{i, j}, B_{i}$ or $C_{i}$ respectively. Hence the sum

$$
\sum_{s \in A_{i, j}} a_{s} D_{i_{1}} \circ \cdots \circ D_{i_{m-1}}\left(P_{s}\right)_{\mid z_{1}=\cdots=z_{2 n}=1},
$$

and the analogous sums for $s \in B_{i}$ or $s \in C_{i}$ respectively are polynomials in $\left(\alpha_{i, j}\right),\left(\beta_{i}\right),\left(\gamma_{i}\right)$ of degree at most $m$. Therefore

$$
D_{i_{1}} \circ \cdots \circ D_{i_{m}}(P)=\sum_{s \in S} a_{s} D_{i_{1}} \circ \cdots \circ D_{i_{m-1}}\left(P_{s}\right)_{\mid z_{1}=\ldots=z_{2 n}=1}
$$

is a polynomial in $\left(\alpha_{i, j}\right),\left(\beta_{i}\right),\left(\gamma_{i}\right)$ of degree at most $m$.

\section{Proof of Theorem 1.1;}

In the following we will show that the integer $A_{(\pi)_{m} \pi^{\prime}}$ of FPLs with link pattern $(\pi)_{m} \pi^{\prime}$ is a polynomial in $m$. Together with [2][Theorem 6.7], which states that $A_{(\pi)_{m} \pi^{\prime}}$ is a polynomial in $m$ with requested degree and leading coefficient for large values of $m$, this proves the theorem.

Denote the corresponding partition of $\pi$ by $\left(\lambda_{1}, \cdots, \lambda_{p}\right)$ and let $q=e^{\frac{2 \pi i}{3}}$ throughout the proof. By Theorem 2.3 the number $A_{(\pi)_{m} \pi^{\prime}}$ is given by

$$
A_{(\pi)_{m} \pi^{\prime}}=\Psi_{(\pi)_{m} \pi^{\prime}}(1, \ldots, 1)
$$

We calculate $\Psi_{(\pi)_{m} \pi^{\prime}}$ in the following three steps:

1. First we calculate $\Psi_{(\pi)_{m+n^{\prime}}}$. The Young diagram corresponding to $(\pi)_{m+n^{\prime}}$ consists of $|\lambda(\pi)|$ boxes which lie on the south-east-diagonals which are indexed by the numbers $m+n^{\prime}+2, \ldots, m+$ $2 n+n^{\prime}-2$. Hence by the recursion in Theorem 2.3 the wheel polynomial $\Psi_{(\pi)_{m+n^{\prime}}}$ is a sum of terms of the form $D_{i_{1}} \circ \cdots \circ D_{i_{k}}\left(\Psi_{()_{m+n+n^{\prime}}}\right)$ - as explained in Remark 2.5 - over $k \leq|\lambda(\pi)|$ and $i_{1}, \ldots, i_{k} \in\left\{m+n^{\prime}+2, \ldots, m+2 n+n^{\prime}-2\right\}$. As mentioned in Remark 2.5, the number of summands and the indices $i_{j}$ of the linear maps $D_{i_{j}}$ in the summands depend only on $\pi$ and not on $m$.

2. Let $\sigma$ be the noncrossing matching of size $m+n+n^{\prime}$ with corresponding integer partition $(\underbrace{m+n, \ldots, m+n}_{n^{\prime} \text { times }}, \lambda_{1}, \ldots, \lambda_{p})$, the Young diagram of $\sigma$ is depictured in Figure 7 Using the third statement of Theorem 2.3 together with $q^{3}=1$ we obtain

$$
\begin{aligned}
\Psi_{\sigma}\left(z_{1}, \ldots, z_{2\left(m+n+n^{\prime}\right)}\right) & =\Psi_{\rho^{-n^{\prime}}\left((\pi)_{\left.m+n^{\prime}\right)}\right.}\left(z_{1}, \ldots, z_{2\left(m+n+n^{\prime}\right)}\right)= \\
& =\Psi_{(\pi)_{m+n^{\prime}}}\left(z_{n^{\prime}+1}, \ldots, z_{2\left(m+n+n^{\prime}\right)}, z_{1}, \ldots, z_{n^{\prime}}\right) .
\end{aligned}
$$

Therefore $\Psi_{\sigma}$ is a sum over terms of the form $D_{i_{1}} \circ \cdots \circ D_{i_{k}}\left(\Psi_{\rho^{-n^{\prime}}()_{m+n+n^{\prime}}}\right)$, where $k \leq|\lambda(\pi)|$ and $i_{1}, \ldots, i_{k} \in\{m+2, \ldots, m+2(n-1)\}$. 


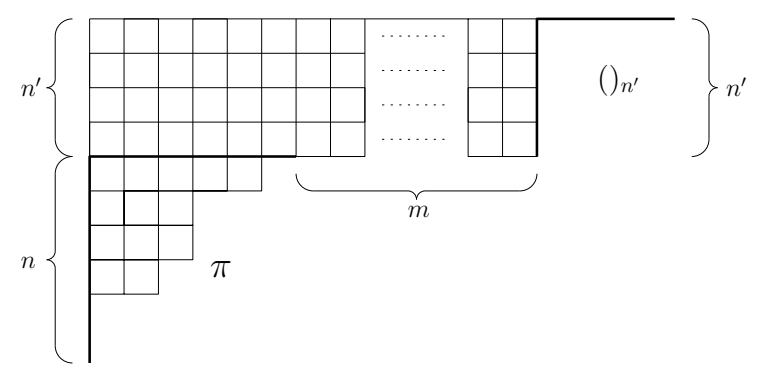

Fig. 7: The Young diagram corresponding to $\sigma$.

3. Since $\sigma<(\pi)_{m} \pi^{\prime}$ and $\sigma$ has just $\left|\lambda\left(\pi^{\prime}\right)\right|$ boxes less than $(\pi)_{m} \pi^{\prime}$, we can calculate $\Psi_{(\pi)_{m} \pi^{\prime}}$ recursively using $\Psi_{\sigma}$ as initial value by Theorem 2.3 and Lemma 2.4 The new boxes in the Young diagram $\lambda\left((\pi)_{m} \pi^{\prime}\right)$ lie on the south-east-diagonals which are indexed by the numbers $2(m+$ $n+1), \ldots, 2\left(m+n+n^{\prime}-1\right)$. Hence, $\Psi_{(\pi)_{m} \pi^{\prime}}$ is a sum of terms of the form $D_{i_{1}} \circ \cdots \circ$ $D_{i_{k^{\prime}}}\left(\Psi_{\rho^{-n^{\prime}}()_{m+n+n^{\prime}}}\right)$ over $k^{\prime} \leq|\lambda(\pi)|+\left|\lambda\left(\pi^{\prime}\right)\right|$. Further the number of summands and the indices $i_{j}$ of the linear maps $D_{i_{j}}$ in the summands depend only on $\pi$ and $\pi^{\prime}$ and not on $m$, compare with Remark 2.5 and Lemma 2.4. By the above considerations and Lemma 2.4, the indices $i_{l}$ for $1 \leq l \leq k^{\prime}$ satisfy $i_{l} \in\left\{m+2, \ldots, m+2(n-1), 2(m+n+1), \ldots, 2\left(m+n+n^{\prime}-1\right)\right\}$.

In order to calculate $A_{(\pi)_{m} \pi^{\prime}}$ we use the identity

$$
A_{(\pi)_{m} \pi^{\prime}}=\Psi_{(\pi)_{m} \pi^{\prime}}(1, \ldots, 1)=\Psi_{\rho^{n^{\prime}}\left((\pi)_{m} \pi^{\prime}\right)}(1, \ldots, 1) .
$$

The second identity is true since $\Psi_{(\pi)_{m} \pi^{\prime}}$ and $\Psi_{\rho^{n^{\prime}}\left((\pi)_{m} \pi^{\prime}\right)}$ just differ by a rotation in the variables $z_{1}, \ldots, z_{2\left(m+n+n^{\prime}\right)}$ by Theorem 2.3 By the above considerations $\Psi_{\rho^{n^{\prime}}\left((\pi)_{m} \pi^{\prime}\right)}$ is a sum of terms of the form $D_{i_{1}} \circ \cdots \circ D_{i_{k^{\prime}}}\left(\Psi_{()_{m+n+n^{\prime}}}\right)$ with $k^{\prime} \leq|\lambda(\pi)|+\left|\lambda\left(\pi^{\prime}\right)\right|$ and $i_{1}, \ldots, i_{k^{\prime}} \in\left\{2, \ldots, n^{\prime}-2, m+n^{\prime}+\right.$ $\left.2, \ldots, m+2 n+n^{\prime}-2,2(m+n)+n^{\prime}+2, \ldots, 2\left(m+n+n^{\prime}\right)\right\}$. Therefore the linear maps $D_{i_{l}}$ which appear in the above summands act trivially on the variables $z_{n^{\prime}+1}, \ldots z_{m+n^{\prime}}, z_{m+2 n+n^{\prime}+1}, \ldots, z_{2\left(m+n+n^{\prime}\right)}$. Denote by $I$ the set of indices of these $z_{i}$. The evaluation of the wheel polynomial $\Psi_{\rho^{n^{\prime}}\left((\pi)_{m} \pi^{\prime}\right) \mid z_{i}=1 \text { for } i \in I}$ is a sum of terms of the form $D_{i_{1}} \circ \cdots \circ D_{i_{k^{\prime}}}\left(\Psi_{()_{m+n+n^{\prime}} \mid z_{i}=1 \text { for } i \in I}\right)=D_{i_{1}} \circ \cdots \circ D_{i_{k^{\prime}}}\left(P\left(\alpha_{i, j}\left|\beta_{i}\right| \gamma_{i}\right)\right)$ where

$$
\begin{aligned}
\alpha_{i, j} & = \begin{cases}1 & i, j \notin I, i<j,\left(j \leq\left(m+n+n^{\prime}\right) \text { or } i>m+n+n^{\prime}\right), \\
0 & \text { otherwise }\end{cases} \\
\beta_{i} & = \begin{cases}m & i \in\left\{m+n^{\prime}+1, \ldots, m+n+n^{\prime}, 2(m+n)+n^{\prime}+1, \ldots, 2\left(m+n+n^{\prime}\right)\right\} \\
0 & \text { otherwise }\end{cases} \\
\gamma_{i} & = \begin{cases}m & i \in\left\{1, \ldots, n^{\prime}, m+n+n^{\prime}+1, \ldots, m+2 n+n^{\prime}\right\} \\
0 & \text { otherwise }\end{cases}
\end{aligned}
$$

By Theorem 3.2 the evaluation $z_{i}=1$ for the remaining variables in the above expressions are polynomials in $\left(\alpha_{i, j}\right),\left(\beta_{i}\right),\left(\gamma_{i}\right)$ of degree at most $k^{\prime} \leq|\lambda(\pi)|+\left|\lambda\left(\pi^{\prime}\right)\right|$. The explicit choice of $\left(\alpha_{i, j}\right),\left(\beta_{i}\right),\left(\gamma_{i}\right)$ 
implies that it is actually a polynomial in $m$ of degree at most $|\lambda(\pi)|+\left|\lambda\left(\pi^{\prime}\right)\right|$. Since $A_{(\pi)_{m} \pi^{\prime}}$ is a sum of these polynomials and the sum is independent of $m$ this proves the theorem.

In the above proof we used the fact that in [2] it was already shown that the number $A_{(\pi)_{m} \pi^{\prime}}$ is a polynomial in $m$ with the correct degree and leading coefficient for large values of $m$. However it seems to be possible to prove the statement about the degree and the leading coefficient by means of wheel polynomials - this is work in progress.

\section{References}

[1] L. Cantini and A. Sportiello. Proof of the Razumov-Stroganov conjecture. J. Combin. Theory, Ser. A, 118(5):1549 - 1574, 2011.

[2] F. Caselli, C. Krattenthaler, B. Lass, and P. Nadeau. On the Number of Fully Packed Loop Configurations with a Fixed Associated Matching. Electronic J. Combin., 11(2), 2004.

[3] J. de Gier and P. Pyatov. Factorised solutions of Temperley-Lieb $q \mathrm{KZ}$ equations on a segment. Adv. Theor. Math. Phys., 14(3):795-878, 2010.

[4] T. Fonseca and P. Zinn-Justin. On some ground state components of the $\mathrm{O}(1)$ loop model. J. Stat. Mech. Theory and Exp., 2009.

[5] A. V. Razumov and Y. G. Stroganov. Combinatorial nature of ground state vector of O(1) loop model. arXiv:0104216, 2001.

[6] D. Romik. Connectivity Patterns in Loop Percolation I: the Rationality Phenomenon and Constant Term Identities. Comm. Math. Phys., 330(2):499-538, 2014.

[7] B. Wieland. A Large Dihedral Symmetry of the Set of Alternating Sign Matrices. Electronic J. Combin., 7, 2000.

[8] D. Zeilberger. Proof of the Alternating Sign Matrix Conjecture. Electronic J. Combin., 3(2), 1996.

[9] P. Zinn-Justin. Six-Vertex, Loop and Tiling models: Integrability and Combinatorics. Habilitation thesis, arXiv:0901.0665, 2009.

[10] P. Zinn-Justin and P. Di Francesco. Quantum Knizhnik-Zamolodchikov equation, totally symmetric self-complementary plane partitions, and alternating sign matrices. Theor. Math. Phys., 154(3):331348, 2008.

[11] J. B. Zuber. On the Counting of Fully Packed Loop Configurations: Some new conjectures. Electronic J. Combin., 11(1), 2004. 